\title{
Free surface simulation of a two-layer fluid by boundary element method
}

\author{
Weoncheol Koo
}

School of Naval Architecture and Ocean Engineering, Univ. of Ulsan, Ulsan, Korea

\begin{abstract}
A two-layer fluid with free surface is simulated in the time domain by a two-dimensional potential-based Numerical Wave Tank (NWT). The developed NWT is based on the boundary element method and a leap-frog time integration scheme. A whole domain scheme including interaction terms between two layers is applied to solve the boundary integral equation. The time histories of surface elevations on both fluid layers in the respective wave modes are verified with analytic results. The amplitude ratios of upper to lower elevation for various density ratios and water depths are also compared.
\end{abstract}

KEY WORDS: Two layer fluid; Internal wave; Time domain; Numerical wave tank; Leap frog integration.

\section{INTRODUCTION}

In the classical view of hydrodynamics calculation, water density is assumed to be uniform and the results came from this premise have been satisfied with most of physical phenomena in the ocean. In some special areas, however, water density is not uniform and two-layer density fluids may exist, caused by changing of the water temperature and salinity. For instance, when warm sea water meets cold water with high salinity, two-density layers can be formed and the internal waves may be generated at the interface between the layers.

The restoring force for water waves, called relative buoyancy force, is proportional to the product of gravity and the density difference between two layers. At the interface between two layers the difference is much smaller than the density difference between air and water. Consequently, internal waves generated at the interface can attain much larger amplitudes than free surface waves. It also takes longer for the restoring force to return water particles to their average positions, and the wave has a period much longer than surface gravity waves (e.g., from 10 minutes to several hours).

The internal waves are considered as a cause of damage of ships and offshore structures due to their huge magnitudes. Several studies related to these unique waves were conducted after the first observation by Russell $(1838,1844)$. Osborne et al. (1978) researched the influence of internal waves on deep sea drilling. Linton and McIver (1995) studied the horizontal cylinders interacted with waves in two-layer fluids. Kuznetsov et al. (2003) also studied wave interaction with a

Corresponding author: Weoncheol Koo

e-mail:wckoo@ulsan.ac.kr
2D floating body in a two-layer fluid. Recently, the influence of local shear flow on the cross flow response of a cylindrical structure was reported by Kim and Rheem (2009).

When two-layer fluids are considered as a two-domain problem, two wave modes are obtained such as surface wave mode (S-mode) and internal wave mode (I-mode). The propagation of internal waves can be predicted by the modified dispersion relation of respective wave mode. Thus, the waves on both fluid surfaces propagate with two different wave numbers $k_{s}$ and $k_{i}$ corresponding to S-mode and I-mode, respectively. The wave number of S-mode $\left(k_{s}\right)$ is smaller than that of I-mode $\left(k_{i}\right)$; thus the propagating waves in S-mode are longer than those in I-mode. The generated waves on free surface and interface in S-mode are in phase and the elevation of free surface is bigger than that of interface. However, in I-mode, the waves are 180 degrees out-of-phase and the free surface amplitudes are usually smaller than the internal waves.

For wave-body interaction in two-layer fluids, Yeung and Nguyen (1999) developed the boundary integral equation method to solve the radiation and diffraction problem for a rectangular barge with finite depth in the frequency domain. Ten and Kashiwagi (2004), Kashiwagi et al. (2006) and Kim and Koo (2010) also studied for a 2D floating body in twolayer fluids with a similar manner.

Even if numerous computational studies of internal waves have been conducted so far, most of calculations are based on frequency domain, assuming of small harmonic amplitudes. However, the internal waves have large amplitudes and long periods, and their interaction with structure may be highly nonlinear. Therefore, a time domain approach has to be required to investigate the nonlinear characteristics of internal waves and their interaction with structures. 
In the present study, a 2-dimensional Numerical Wave Tank (NWT) technique based on a two-domain Boundary Element Method in the time domain is developed to simulate internal waves in two-layer fluids. The computational domain is assumed to be filled with potential fluids of two different densities and a flat sea bottom.

The boundary integral equation transformed from the governing equation of both fluid domains is solved by using the whole domain scheme to satisfy the interface condition of two computational domains. The leap-frog time marching scheme is newly developed for time integration of surface boundary conditions on both domains. The time-varying waves on both surfaces are measured to investigate the characteristics of internal waves for various ratios of fluid density and water depth.

The present simulation is based on the leap-frog time integration scheme and this time-domain method can be easily extended to fully nonlinear time simulation as changing the instantaneous surface boundaries with updating the node locations. Therefore, the present work is a worthwhile study for solving the fully nonlinear time domain solution of two-layer fluid in the next study.

Since the stratification of two-density fluids is assumed to be stable, the present calculation condition is based that no fluid-mixing occurs at the interface during the time simulation.

\section{MATHEMATICAL FORMULATION}

\section{Governing Equation and Boundary Integral Equation}

The computational domain composed of two densityfluids is assumed to be filled with inviscid, incompressible and irrotational fluids. Assuming that the water particle has a harmonic motion of angular frequency $\omega$, the velocity potential is defined as Eq. (1):

$$
\phi(\vec{x}, t)=\operatorname{Re}\left[\Phi(\vec{x}) e^{-i \omega t}\right]
$$

The gradient of the velocity potential can express water particle velocity, and the continuous equation is satisfied in the computational domain, thus the Laplace equation can be used as a governing equation.

$\nabla^{2} \phi^{(m)}=0$

where $\mathrm{m}=1,2$ denote upper and lower fluid domains, respectively and surface elevation on each boundary is also given as:

$\eta^{(m)}(x, t)=a_{0}^{(m)} \cos (k x-\omega t)$

where $a_{0}^{(m)}$ is wave amplitude on respective fluid surfaces.

Fig. 1 shows the entire computational domain with symbols of parameters.

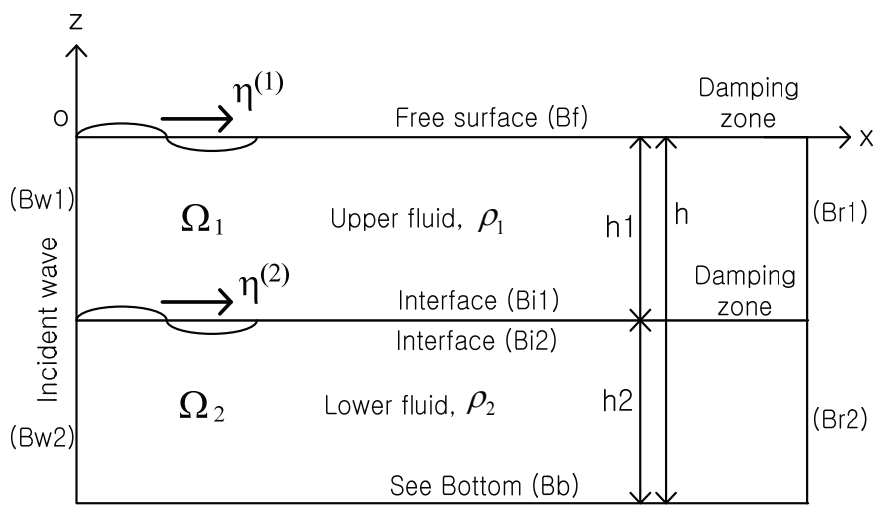

Fig. 1 Computational domain of two layered fluids.

Using the Green function satisfying the Laplace equation, the governing equation can be transformed to a boundary integral equation (Eq. (4)).

$\alpha \phi_{i}^{(m)}=\iint_{\Omega_{(m)}}\left(G_{i j}^{(m)} \frac{\partial \phi_{j}^{(m)}}{\partial n}-\phi_{j}^{(m)} \frac{\partial G_{i j}^{(m)}}{\partial n}\right) d s$

where the Green function is $G_{i j}\left(x_{i}, z_{i}, x_{j}, z_{j}\right)=-(1 / 2 \pi) \ln R_{1}$ for a two-dimensional problem in each domain $\left(\Omega_{1}, \Omega_{2}\right), \alpha$ is a solid angle (it is 0.5 when singularities are on the boundary) and $R_{1}$ is the distance between source $\left(x_{i}, z_{i}\right)$ and field points $\left(x_{j}, z_{j}\right)$. It is noted that the source and field points to obtain the Green function in the present study were defined only in the same fluid domain, i.e. the Green function for the upper domain $\left(G_{i j}^{(1)}\right)$ is obtained from the collocation points only in the upper domain.

\section{Boundary Conditions}

In order to solve the boundary integral equation the corresponding boundary conditions for both fluid domains should be defined which include free surface, interface, rigid end-wall, sea bottom, and incident wave boundaries.

Linearized dynamic and kinematic free surface boundary conditions are described as:

$\frac{\partial \phi}{\partial t}=-g \eta^{(1)}-\frac{P}{\rho} \quad$ on $\quad z=0$

$\frac{\partial \eta^{(1)}}{\partial t}=\frac{\partial \phi^{(1)}}{\partial z} \quad$ on $\quad z=0$

where $g$ is gravity and air pressure $(P)$ on the free surface is set to be zero.

The linearized dynamic condition on the interface is related to the density ratio of upper to lower fluids, and the water particle velocity on the interface is continuous, thus the dynamic and kinematic interface boundary conditions are given as: 
$\rho_{1}\left(\frac{\partial \phi^{(1)}}{\partial t}+g \eta^{(2)}\right)=\rho_{2}\left(\frac{\partial \phi^{(2)}}{\partial t}+g \eta^{(2)}\right)$ on $z=-\mathrm{h} 1$

$\frac{\partial \phi^{(1)}}{\partial z}=\frac{\partial \phi^{(2)}}{\partial z}=\frac{\partial \eta^{(2)}}{\partial t} \quad$ on $z=-\mathrm{h} 1$

It is noted that the normal velocities of upper and lower domains on the interface are in the opposite direction, e.g., $\partial \phi^{(1)} / \partial n=\partial \phi^{(2)} / \partial n$.

A rigid sea bottom as a no-penetration condition is described as in the lower fluid domain:

$\frac{\partial \phi^{(2)}}{\partial z}=0 \quad$ on $\quad z=-\mathrm{h}$

No-normal flux (no-penetration) condition can also be applied to vertical right-end walls in both domains $\left(\partial \phi^{(1)} / \partial n\right.$ $\left.=0, \partial \phi^{(2)} / \partial n=0\right)$.

\section{Incident wave potential and Dispersion relation}

The incident waves are obtained by solving the governing equation with boundary conditions in two-layer fluid domains, and are expressed as follows (Yeung and Nguyen, 1999):

Upper domain

$$
\begin{aligned}
\phi^{(1)}= & \frac{-a_{0}^{(1)} \cdot c h 1}{\omega k}\left[\left(\omega^{2} \cdot t h 1-g k\right) \cosh k(z+h 1)\right. \\
& \left.-\left(\omega^{2}-g k \cdot t h 1\right) \sinh k(z+h 1)\right] \sin (k x-\omega t)
\end{aligned}
$$

Lower domain

$$
\begin{aligned}
\phi^{(2)}= & \frac{a_{0}^{(1)} \cdot \operatorname{ch} 1}{\omega k \cdot \operatorname{sh} 2}\left(\omega^{2}-g k \cdot t h 1\right) \cosh k(z+h 2) \\
& \times \sin (k x-\omega t)
\end{aligned}
$$

where $a_{0}^{(1)}=$ incident wave amplitude on the free surface, $\operatorname{ch} 1=\cosh (k \mathrm{~h} 1), \operatorname{sh} 2=\sinh (k \mathrm{~h} 2)$ and $\operatorname{th} 1=\tanh (k \mathrm{~h} 1)$. Note that the amplitude of incident wave was selected as a constant value for all calculations $(0.1 \mathrm{~m}$ in this study). Wave number $(k)$ applied to Eqs. (10) and (11) can be obtained using the following dispersion relations for each wave mode.

Water wave dispersion relations of surface and internal wave modes in two-layer fluid domains are given respectively as (Yeung and Nguyen, 1999):

Surface wave mode (S-mode)

$$
\begin{aligned}
\omega^{2} & =\frac{g k}{2(1+\gamma t h 1 \cdot t h 2)} \\
& \times\left(t h 1+t h 2+\sqrt{(t h 1+t h 2)^{2}-4(1-\gamma) t h 1 \cdot t h 2(1+\gamma t h 1 \cdot t h 2)}\right)
\end{aligned}
$$

Internal wave mode (I-mode)

$$
\begin{aligned}
& \omega^{2}=\frac{g k}{2(1+\gamma t h 1 \cdot t h 2)} \\
& \times\left(t h 1+t h 2-\sqrt{(t h 1+t h 2)^{2}-4(1-\gamma) t h 1 \cdot t h 2(1+\gamma t h 1 \cdot t h 2)}\right)
\end{aligned}
$$

where $\gamma=\rho_{1} / \rho_{2}, \operatorname{th} 1=\tanh (k \mathrm{~h} 1)$ and $t h 2=\tanh (k \mathrm{~h} 2)$. With given wave frequency, the wave number obtained by Eq. (12) is $k_{s}$ and the number by Eq. (13) is $k_{I}$. Therefore, two different wave numbers are generated with one given frequency depending on wave mode.

\section{Wave Amplitude Ratio}

The ratio of free surface wave amplitude to interface amplitude can be determined by Eqs. (3), (8) and (11).

$\frac{a_{0}^{(2)}}{a_{0}^{(1)}}=\cosh k h 1\left(1-\frac{g k}{\omega^{2}} \tanh k h 1\right)$

\section{Time Marching (Leap-frog method)}

In order to calculate the time-varying wave elevation and velocity potential on the boundaries the free surface and interface boundary conditions shown in Eqs. (5) to (8) must be integrated with respect to time. In the present study a leapfrog method is adapted as a time integration scheme to obtain a time histories of wave elevation and velocity potential on both surfaces. The leap-frog integration method is equivalent to calculating the position of boundary surface and the velocity potential at interleaved time points, so that they 'leapfrog' over each other. For example, the position is known at integer plus a half time step and then the velocity potential can be obtained at integer plus the next time step. Therefore, the time marching for free surface is as follows:

Free surface

$$
\begin{aligned}
& \left(\eta^{(1)}\right)^{t+\frac{\Delta t}{2}}=\left(\eta^{(1)}\right)^{t-\frac{\Delta t}{2}}+\Delta t\left(\frac{\partial \phi^{(1)}}{\partial z}\right)^{t} \text { on } z=0 \\
& \left(\phi^{(1)}\right)^{t+\Delta t}=\left(\phi^{(1)}\right)^{t}-\Delta t \cdot g\left(\eta^{(1)}\right)^{t+\frac{\Delta t}{2}} \text { on } z=0
\end{aligned}
$$

Artificial damping zone on free surface

$$
\begin{aligned}
& \left(\eta^{(1)}\right)^{t+\frac{\Delta}{2}}=\left(\eta^{(1)}\right)^{t \frac{\Delta t}{2}}+\Delta t\left(\frac{\partial \phi^{(1)}}{\partial z}\right)^{t}-\Delta t \cdot \mu_{2}\left(\eta^{(1)}\right)^{t \frac{\Delta t}{2}} \text { on } z=0 \\
& \left(\phi^{(1)}\right)^{t+\Delta t}=\left(\phi^{(1)}\right)^{t}-\Delta t \cdot g\left(\eta^{(1)}\right)^{t+\frac{\Delta}{2}}-\Delta t \cdot \mu_{1}\left(\frac{\partial \phi^{(1)}}{\partial n}\right)^{t} \text { on } z=0
\end{aligned}
$$

where $\frac{\partial \phi^{(1)}}{\partial z}=\frac{\partial \phi^{(1)}}{\partial n}$ on $z=0$ and 


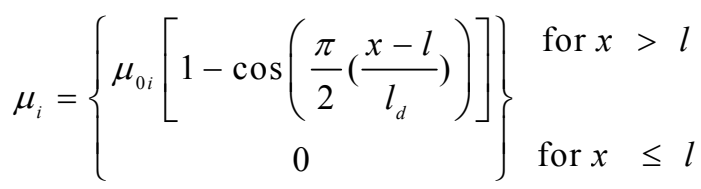

$l$ is the length of fluid domain without damping zone and $l_{d}$ is the length of damping zone. The optimum $l_{d}$ was chosen greater than 2 incident wavelengths. The performance and efficiency of the artificial damping scheme was numerically confirmed in the next chapter. Through linear stability analysis, the relation of damping coefficients $\mu_{01}$ and $\mu_{02}$ were obtained, i.e. $\mu_{02}=k \mu_{01}$ is used to minimize the dispersion error at the discontinuity of boundary conditions. In this study, $\mu_{01}$ of 3.5 was chosen as an optimized damping coefficient after comprehensive numerical tests with given inputs.

The time marching for interface boundary

$$
\begin{array}{rlrl}
\left(\eta^{(2)}\right)^{t+\frac{\Delta t}{2}}=\left(\eta^{(2)}\right)^{t-\frac{\Delta t}{2}}+\Delta t\left(\frac{\partial \phi^{(2)}}{\partial z}\right)^{t} & \text { on } z=-\mathrm{h} 1 \\
\left(\phi^{(2)}-\gamma \phi^{(1)}\right)^{t+\Delta t} & =\left(\phi^{(2)}-\gamma \phi^{(1)}\right)^{t} \\
& +\Delta t(\gamma-1) \cdot g\left(\eta^{(2)}\right)^{t+\frac{\Delta t}{2}} & \text { on } z=-\mathrm{h} 1
\end{array}
$$

Artificial damping zone on interface

$$
\begin{aligned}
& \left(\eta^{(2)}\right)^{t+\frac{\Delta t}{2}}=\left(\eta^{(2)}\right)^{t-\frac{\Delta t}{2}}+\Delta t\left(\frac{\partial \phi^{(2)}}{\partial z}\right)^{t}-\Delta t \cdot \mu_{2}\left(\eta^{(2)}\right)^{t-\frac{\Delta t}{2}} \\
& \left(\phi^{(2)}-\gamma \phi^{(1)}\right)^{t+\Delta t}=\left(\phi^{(2)}-\gamma \phi^{(1)}\right)^{t} \\
& +\Delta t(\gamma-1) \cdot g\left(\eta^{(2)}\right)^{t+\frac{\Delta t}{2}}-\Delta t \cdot \mu_{1}\left(\frac{\partial \phi^{(2)}}{\partial n}\right)^{t} \\
& \text { where } \frac{\partial \phi^{(2)}}{\partial z}=\frac{\partial \phi^{(2)}}{\partial n} \text { on } z=-\mathrm{h} 1
\end{aligned}
$$

\section{NUMERICAL RESULTS AND DISCUSSION}

Surface elevation on free surface and interface in a twolayer fluid domain can be obtained at every time step using the leap-frog time integration of surface boundary conditions. In order to validate the time marching (time integration) scheme and verify the calculated elevations convergence tests for the number of node and computational time interval were performed. When the number of nodes on free surface per wavelength is greater than 38 , the surface elevations converge well in S-mode at the time step smaller than $d t=T$ / 64. In I-mode, the elevations converge after the node number is greater than 16. In order to damp out the incident wave energy efficiently the optimum length of artificial damping zone has to be chosen. In this study, the damping zone should be greater than two wave lengths from the convergence test shown in Fig. 2. Based on these convergence tests, all the results in the current study are calculated with the selected computational parameters.

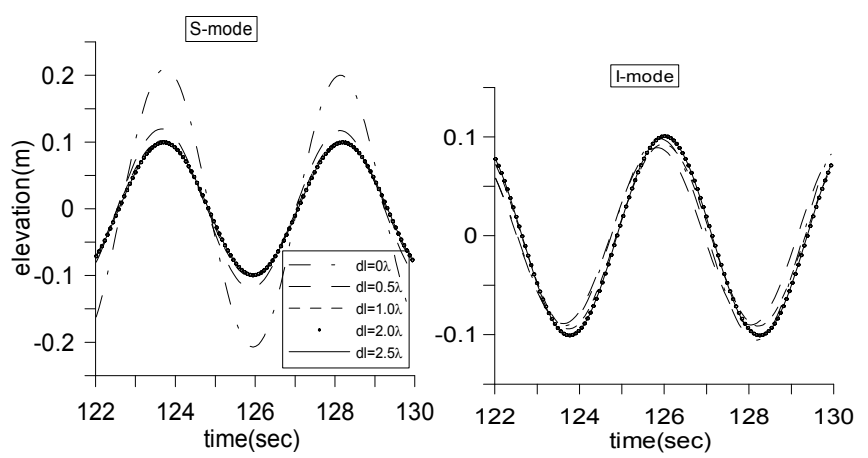

Fig. 2 Convergence test for the length of damping zone (free surface elevation).

Fig. 3 shows a comparison of wave elevation on both fluid surfaces with a given computational condition. In Smode, the free surface elevation is much greater than interface elevation and no phase difference is found. In Imode, however, the interface elevation is relatively high compared to the free surface elevation and the phase difference is 180 degrees.

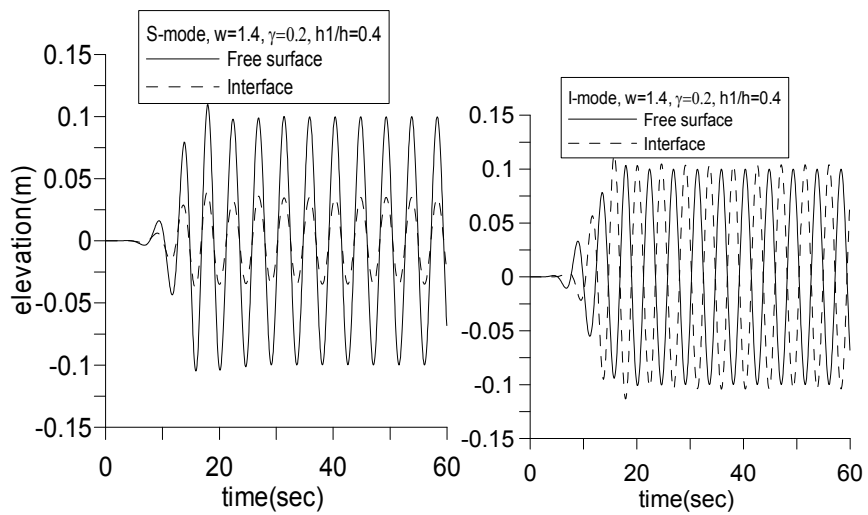

Fig. 3 Comparison of wave elevation at each boundary, $\mathrm{h}=10 \mathrm{~m}$.
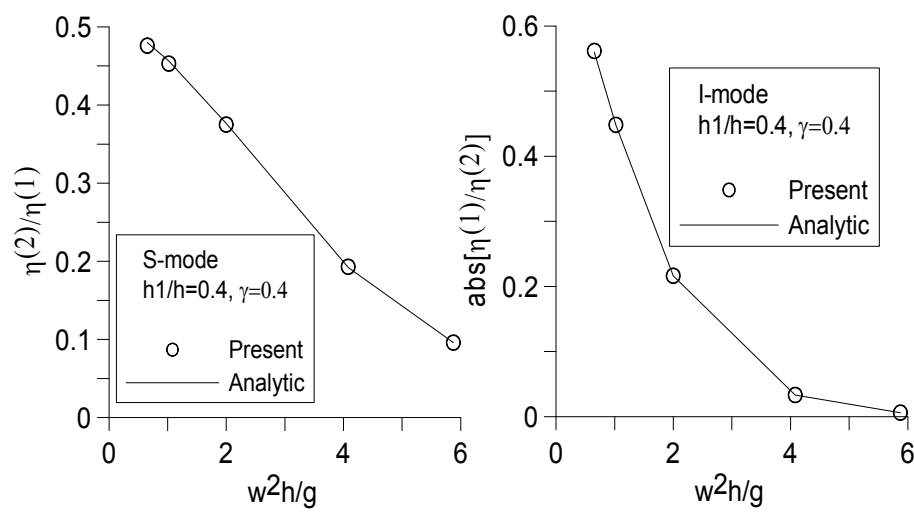

Fig. 4 Comparison of amplitude ratio of the present results with analytic solutions, $\mathrm{h}=10 \mathrm{~m}$. 
The amplitude ratio of free surface to interface is compared in Fig. 4. The analytic solution is calculated by Eq. (14), while the numerical result is measured at each surface boundary. Both results are well agreed in both wave modes. As wave frequency increases, the relative magnitude of interface elevation compared to free surface elevation decreases in S-mode, while increases in I-mode.

Fig. 5 shows a variation of the amplitude ratios for three different density ratios. As fluid density ratio decreases (density difference is large), the interface elevation in Smode is relatively small especially in long wave region and the difference reduces with high wave frequencies. In I-mode, the interface elevation relatively increases in long wave region as fluid density ratio increases. In other words, the maximum internal wave can be generated in I-mode with a long period incident wave and small density different fluids.

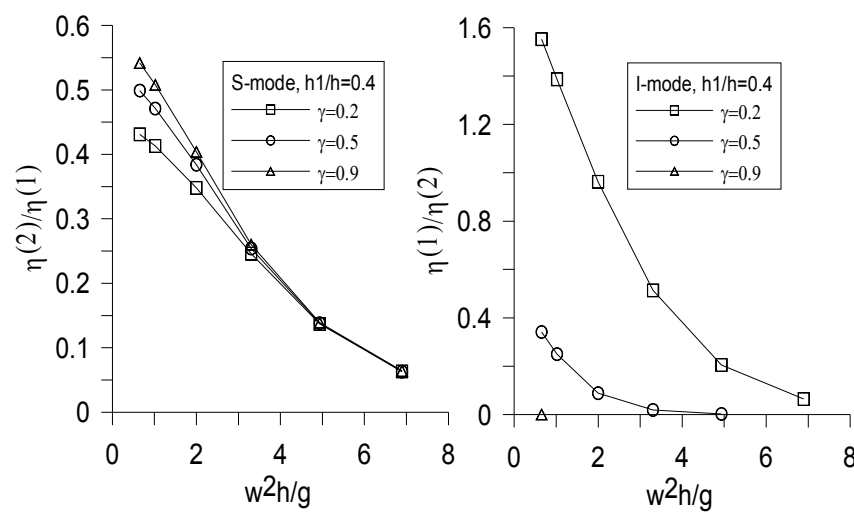

Fig. 5 Comparison of wave amplitude ratio for various fluid density ratios, $\mathrm{h}=10 \mathrm{~m}$.

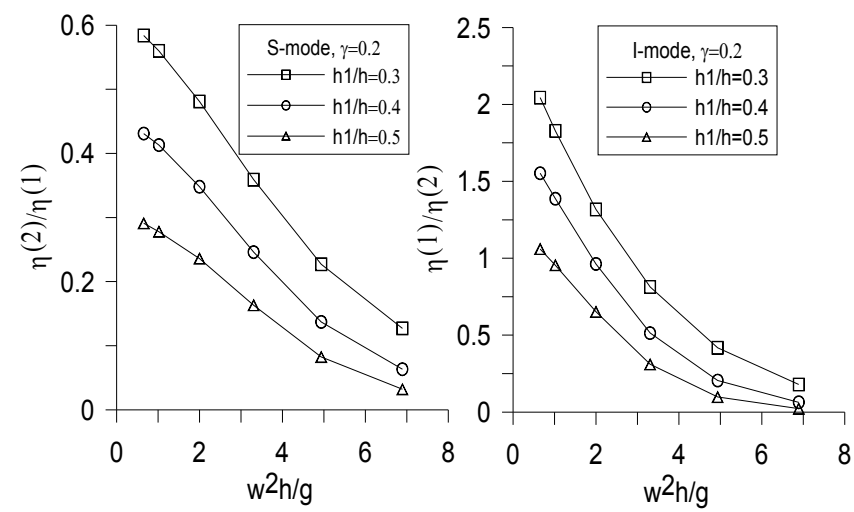

Fig. 6 Comparison of wave amplitude ratio for various water depth ratios, $\mathrm{h}=10 \mathrm{~m}$.

In order to investigate the effect of water depth the wave amplitude ratio for various water-depth ratios are compared in Fig. 6. Since the internal wave amplitude is related to the incident wave amplitude in S-mode and the velocity profile decreases from free surface to the sea bottom, the interface wave amplitude decreases as upper water depth increases. However, the wave amplitude on interface rather increases in I-mode.

\section{CONCLUSIONS}

Wave elevations on free surface and interface in twodensity fluid domains were simulated in the time domain by a 2-dimensional potential-flow-based Numerical Wave Tank. The leap-frog time marching scheme was newly developed for time integration of surface boundary conditions on both computational domains. A whole domain scheme was used for solving the boundary integral equation of two fluid domains. Artificial damping zone was located near the end of both fluid surfaces to absorb the wave energy propagating from incident boundaries. Convergence tests for various input parameters were performed to select the optimum values of computational setup and the simulated waves of respective wave modes were well agreed with analytic results.

The wave amplitude ratios of upper and lower fluid surfaces were calculated for various fluid density ratios and water depths to investigate the characteristics of internal waves.

\section{REFERENCES}

Kashiwagi, M. Ten, I. and Yasunaga, M., 2006. Hydrodynamics of a body floating in a two-layer fluid of finite depth. Part 2. Diffraction problem and waveinduced motions. Journal of Marine Science and Technology, 11, pp. 150-164.

Kim, M.G. and Koo, W.C., 2010. Numerical Analysis of Hydrodynamic Forces on a Floating Body in Two-layer Fluids. Journal of the Society of Naval Architects of Korea, 47(3), pp. 369-376.

Kim, Y.-C. and Rheem, C., 2009. Cross flow response of a cylindrical structure under local shear flow. International Journal of Naval Architecture and Ocean Engineering, 1(2), pp. 101-107.

Kuznetsov, N. McIver, M. and McIver, P., 2003. Wave interaction with two-dimensional bodies floating in a two-layer fluid: uniqueness and trapped modes. Journal of Fluid Mechanics, 490, pp. 321-331

Lintom, C.M. and McIver, M., 1995. The interaction of waves with horizontal cylinders in two-layer fluids. Journal of Fluid Mechanics, 304, pp. 213-219.

Osborne, A.R. and Burch, T.L., 1978. The influence of internal waves on Deep-Water Drilling. Journal of Petroleum Technology, 30(10), pp. 1497-1504.

Russell, J.S., 1838. Report to committee on waves. British Association of the Advancement of Science, Liverpool, UK, pp. 417-496.

Russell, J.S., 1844. Report to committee on waves. British Association of the Advancement of Science, York, UK, pp. 311-390.

Ten, I. and Kashiwagi, M., 2004. Hydrodynamics of a body floating in a two-layer fluid of finite depth. Part 1. Radiation problem. J Ma Sci Technology, 9, pp. 127-141.

Yeung, R.W. and Nguyen, T., 1999. Radiation and diffraction of waves in a two-layer fluid. Proc. of the $22^{\text {nd }}$ Symposium of Naval Hydrodynamics, Washington, DC, pp. 875-891. 